\title{
Proton Disorder and Superionicity in Hot Dense Ammonia Ice
}

\author{
S. Ninet, F. Datchi, and A. M. Saitta \\ Institut de Minéralogie et de Physique des Milieux Condensés, Université Pierre et Marie Curie - Paris 6, \\ CNRS UMR 7590, Paris, France \\ (Received 16 January 2012; published 19 April 2012)
}

\begin{abstract}
We report the experimental discovery of a new phase of ammonia ice, stable at pressures above $57 \mathrm{GPa}$ and temperatures above $700 \mathrm{~K}$. The combination of our experimental results and $a b$ initio molecular dynamics simulations reveal that this new phase is a superionic conductor, characterized by a large proton diffusion coefficient $\left(1.0 \times 10^{-4} \mathrm{~cm}^{2} / \mathrm{s}\right.$ at $\left.70 \mathrm{GPa}, 850 \mathrm{~K}\right)$. Proton diffusion occurs via a Grotthuss-like mechanism, at a surprisingly lower temperature than in water ice. This may have implications for the onset of superionicity in the molecular ice mixtures present in Jovian planets. Our simulations further suggest that the anisotropic proton hopping along different $\mathrm{H}$ bonds in the molecular solid may explain the formation of the recently predicted ionic phase at low temperatures.
\end{abstract}

PACS numbers: 64.70.kt, 61.50.Ks, 62.50.-p, 66.30.jp

At ambient pressure, solid ammonia is a molecular crystal where strong covalent bonds coexist with weaker hydrogen bonds (HBs). Recent density functional theory calculations [1] predict that $\mathrm{NH}_{3}$ transforms above $90 \mathrm{GPa}$, at $0 \mathrm{~K}$, into an ionic solid consisting of layers of $\mathrm{NH}_{4}^{+}$and $\mathrm{NH}_{2}^{-}$. Yet, no experimental evidence of this phase has been reported until now. Previously, high-temperature ab initio molecular dynamics simulations predicted the existence of a superionic state in both $\mathrm{H}_{2} \mathrm{O}$ and $\mathrm{NH}_{3}$ systems (at $P \gtrsim 60 \mathrm{GPa}$ and $T \gtrsim 1200 \mathrm{~K}$ in $\mathrm{NH}_{3}$ ) [2], characterized by a fast diffusion of protons across the fixed $\mathrm{N}$ or $\mathrm{O}$ lattice. This prediction has stimulated several experiments, mainly focused on the measurements of the water melting curve because a change of slope is expected at the onset of the transition. A kink on the melting line of water was detected by different groups [3-5] but not at the same $P-T$ conditions. The exact location of the superionic phase is primordial, as it determines whether the Neptune and Uranus isentropes cross the superionic region. Unlike water, high $P-T$ data on $\mathrm{NH}_{3}$ are very scarce and essentially limited to shock wave measurements in the fluid phase [6-8]. In this Letter, we report a detailed experimental and theoretical investigation of the phase diagram of $\mathrm{NH}_{3}$ up to $100 \mathrm{GPa}$ and $850 \mathrm{~K}$. It shows the presence of a new protondisordered phase above $57 \mathrm{GPa}$ and $700 \mathrm{~K}$. The combination of our experimental results with ab initio molecular dynamics simulations enables us to establish that this new phase exhibits superionic proton conduction.

The experimental phase diagram of ammonia displays five solid phases. At low temperatures $(T)$ and ambient pressure $(P)$, ammonia crystallizes in the proton-ordered cubic $P 2{ }_{1} 3$ phase I, where each molecule forms six equivalent weak HBs, bent at $160^{\circ}$. By compressing the liquid above $\sim 220 \mathrm{~K}$, a rotationally disordered (plastic) phase II with hexagonal close-packed (hcp) structure is obtained. Compression above $\sim 260 \mathrm{~K}$ gives another plastic phase, denoted III, with face-centered cubic (fcc) structure. All those phases transform at higher pressures $(3.6 \mathrm{GPa}$ at $300 \mathrm{~K}$ ) to the proton-ordered solid IV whose structure is orthorhombic $\left(P 2_{1} 2_{1} 2_{1}\right)$ [9-11]. The nitrogen lattice in phase IV is very close (within $0.11 \AA$ ) to the hcp lattice. The topology of the HBs is more complex than in phase I, since there are three types of HBs with different geometries and strengths $[9,12]$. This anisotropy increases at the transition to the isostructural phase $\mathrm{V}$ at $12 \mathrm{GPa}-300 \mathrm{~K}$ in $\mathrm{NH}_{3}$ (18 GPa in $\mathrm{ND}_{3}$ ), since the strongest $\mathrm{HB}$, which links molecules across $(a, b)$ planes, becomes nearly linear whereas the other two remain bent $[11,13]$.

In this work, we studied $\mathrm{NH}_{3}$ samples loaded in diamond anvil cells (see Experimental Methods in Ref. [14]). To locate the transition between the plastic (III) and ordered (V) crystalline phases, we used the change in shape of the stretching $\nu_{1}-\nu_{3}$ Raman band, as well as the presence (respectively, absence) of lattice modes in phase V (respectively, phase III). Our experimental data are presented in the phase diagram depicted in Fig. 1. Two regimes are observed: (i) Up to $57 \mathrm{GPa}$, the transition temperature $\left(T_{c}\right)$ steadily increases with pressure, and the new transition points closely follow the extrapolation of our previous data [15] to $20 \mathrm{GPa}$; (ii) above $57 \mathrm{GPa}$, the increase rate of $T_{c}$ abruptly drops, and the transition points fall on a line of slope $1.2(3) \mathrm{K} / \mathrm{GPa}$. This kink on the transition line strongly suggests the presence of a new phase, denoted $\alpha$, above $57 \mathrm{GPa}$ and $710 \mathrm{~K}$. The Raman spectra [Fig. 2(a)] show that the $\alpha$ phase is proton-disordered, since the $\nu_{3}$ band is weak, very large, and unstructured. No lattice mode could be detected, analogously to the disordered phase III.

Using x-ray diffraction, we observed that annealing phase $\mathrm{V}$ at $16.5 \mathrm{GPa}$ produced the fcc phase III at $472 \mathrm{~K}$ as expected. Between 25 and $55 \mathrm{GPa}$, and temperatures above the transition line, the $\mathrm{x}$-ray patterns are characteristic of either the fcc or hcp structure, depending on whether the solid is obtained by compressing phase III or annealing phase $\mathrm{V}$, respectively. This is easily understood 


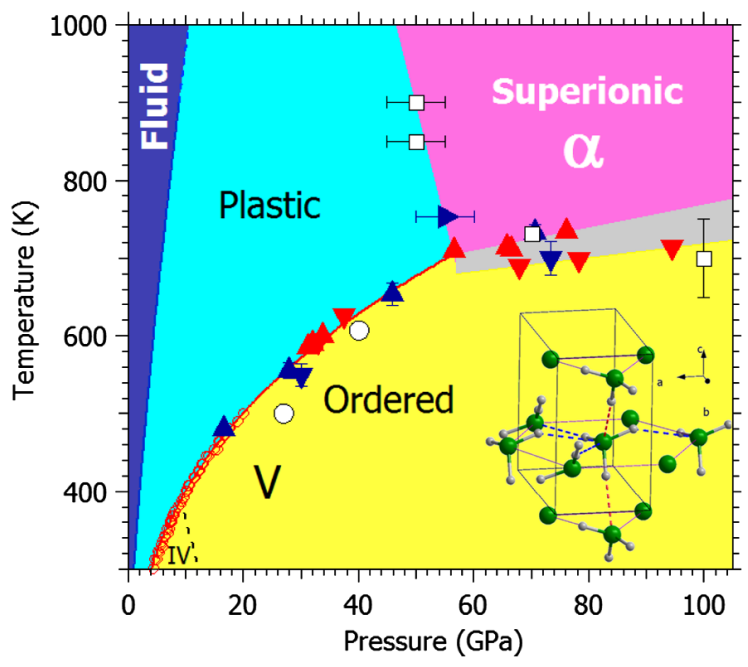

FIG. 1 (color online). Phase diagram of ammonia. Color symbols: Our experimental data. Open red circles and the red solid line: Our previous Raman data and their fit with a Simon-type equation, respectively [15]. Red and blue symbols: New data from Raman scattering and x-ray diffraction experiments, respectively. Up and down triangles: Annealing and cooling runs, respectively. White symbols, our calculations; circles, orientational order-disorder transition points; squares, transition points to the diffusive superionic phase. The structure of phase $\mathrm{V}$ is depicted, with emphasis on the pseudo-hcp $A B A$ stacking. $\mathrm{H}$ bonds are shown as color dashed lines, distinguishing those linking molecules in the same plane (blue) from those linking molecules across the planes (red).

if one considers that the fcc and hcp structures are very close in energy [15], but going from one to the other implies large diffusion of atoms, which is increasingly difficult at high pressure.

The collected x-ray patterns on heating phase $\mathrm{V}$ at $70.6 \mathrm{GPa}$ are shown in Fig. 2(b) (see also Ref. [14]). The $\mathrm{V}-\alpha$ phase transition is revealed by a discontinuous jump of the $c$ axis length at 743(10) K [Fig. 2(c)] and the disappearance of weak reflections such as (120). The doublet nature of the reflections at 2.05 and $2.32 \AA$, characteristic of the orthorhombic structure, is still observed above the transition, and the refinement of the x-ray pattern shows that the $\alpha$ phase is better fitted with the orthorhombic rather than the hcp structure (for details about the refinement, see Ref. [14]). Combining our experimental data for the volume jump across the transition with the Clapeyron equation, we evaluate the entropy variation $\Delta S$ along the transition line. As seen in Fig. 2(d), $\Delta S$ is nearly constant at about 1.0(4) $R$ below $57 \mathrm{GPa}$ but strongly increases at the $\mathrm{V}-\alpha$ transition at $70 \mathrm{GPa}$ to $4(2) R$. This suggests that the number of accessible sites for the $\mathrm{H}$ atoms is greater in the $\alpha$ phase than in the plastic phases. A more quantitative analysis of $\Delta S$ will be given below, in light of our theoretical results. In another run, we compressed the fcc phase III from 20 to $100 \mathrm{GPa}$ at the constant temperature of $750 \mathrm{~K}$. We observed that the cubic unit cell is conserved up to $100 \mathrm{GPa}$, that is, above the expected transition line from III to $\alpha$. Within experimental accuracy, we could not detect any discontinuity in the equation of state. Nonetheless, a sudden change of compressibility clearly occurs around $55 \mathrm{GPa}$, which we attribute to the transition between phase III and $\alpha$ [Fig. 2(e)]. It thus appears that the $\alpha$ phase can either have a pseudo-hcp or a fcc $N$ lattice depending on the followed thermodynamic path. This confirms that $\mathrm{H}$ atoms drive this phase transition, since the $N$ lattice remains fixed.

To obtain a microscopic description of the $\alpha$ phase, we performed ab initio molecular dynamics simulations along several thermodynamic paths. The complete description of theoretical methods is given in the Supplemental Material [14]. We first studied the order-plastic transition for $P<57 \mathrm{GPa}$. On heating phase V at $27 \mathrm{GPa}$, we detected that the orientational order-disorder transition occurs at $500 \mathrm{~K}$ with stepwise rotations of $\mathrm{H}$ atoms about their molecular axis, while full molecular rotations are observed above $700 \mathrm{~K}$. This confirms that the hightemperature solid is plastic at this pressure. Similarly, we heated up the ordered cubic phase I at $40 \mathrm{GPa}$; in this case, we directly observed full molecular rotations above $600 \mathrm{~K}$ [Fig. 3(a)]. At these two pressures, no molecular dissociation was observed up to $1200 \mathrm{~K}$. As shown in Fig. 1, these order-disorder transition points are in good agreement with experiments.

When heating phase $\mathrm{V}$ at $70 \mathrm{GPa}$, we never crossed the plastic phase [see Fig. 3(a)]. Instead, we detected occasional proton jumps along hydrogen bonds, resulting in the instantaneous formation of $\left(\mathrm{NH}_{4}^{+}, \mathrm{NH}_{2}^{-}\right)$ionic pairs. The temperature at which this process starts depends on the specific HB. Indeed, we can distinguish HB-linking molecules located in the same $(a, b)$ plane, denoted $\mathrm{HB}_{a b}$, from those linking molecules across different planes, denoted $\mathrm{HB}_{c}$. The fraction of proton jumps as a function of temperature at $70 \mathrm{GPa}$ for the two $\mathrm{HB}$ types is presented in Fig. 3(b): Jumps along $\mathrm{HB}_{c}$ bonds, i.e., across molecular planes, start at a lower temperature $(T \sim 600 \mathrm{~K})$ than those within the molecular plane along $\mathrm{HB}_{a b}$ bonds $(T \sim$ $740 \mathrm{~K})$. Below $740 \mathrm{~K}$, the $\left(\mathrm{NH}_{4}^{+}, \mathrm{NH}_{2}^{-}\right)$pairs are very short-lived $(\sim 10 \mathrm{fs})$, and protons eventually jump back to their parent molecules. Beyond $740 \mathrm{~K}$, together with the first in-plane jumps, an abrupt increase of jumps along the $c$ axis is observed. At this stage, proton diffusion sets in across the crystal, as revealed by the sudden increase of the mean square displacement of protons [see Fig. 3(b)]: Proton jumps along any of the HBs become possible, and diffusion may thus occur through a Grotthuss-like mechanism [16]. The calculated proton diffusion coefficient at $70 \mathrm{GPa}$ equals $0.25 \times 10^{-4} \mathrm{~cm}^{2} / \mathrm{s}$ at $750 \mathrm{~K}$, $1.0 \times 10^{-4} \mathrm{~cm}^{2} / \mathrm{s}$ at $850 \mathrm{~K}$, and $2.2 \times 10^{-4} \mathrm{~cm}^{2} / \mathrm{s}$ at $1200 \mathrm{~K}$. These values are similar to the one reported [2] at $150 \mathrm{GPa}$ and $2000 \mathrm{~K}\left(3 \pm 0.4 \times 10^{-4} \mathrm{~cm}^{2} / \mathrm{s}\right)$. For comparison, the diffusion coefficient of protons is 

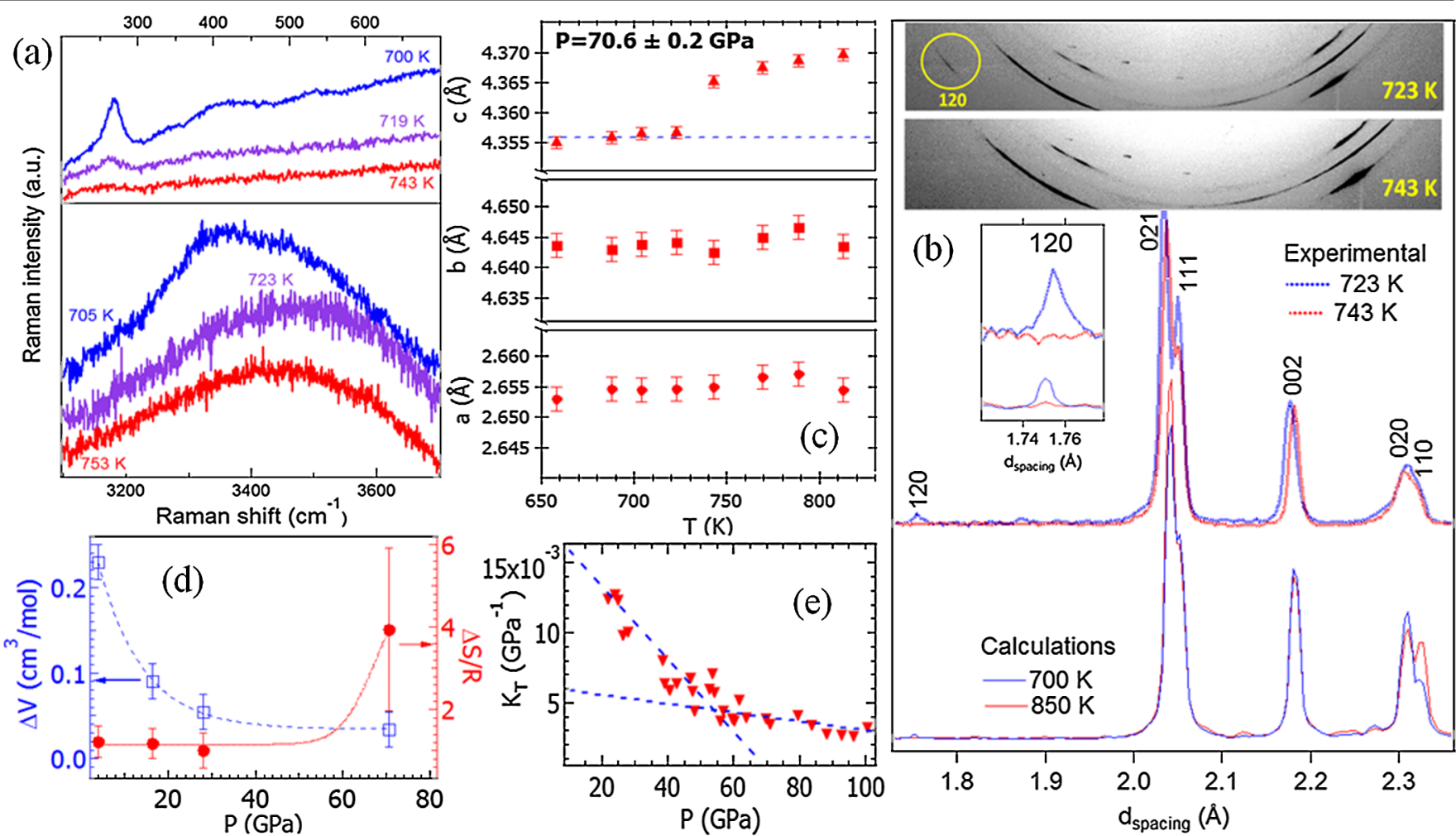

FIG. 2 (color online). (a) Raman spectra of $\mathrm{NH}_{3}$ across the V- $\alpha$ transition: lattice modes at 62 GPa (top) and stretching modes at $93 \mathrm{GPa}$ (bottom). (b) X-ray images (top) and integrated patterns (bottom) at $70.6 \mathrm{GPa}$, below (723 K) and above (743 K) the V- $\alpha$ transition $T$. Only the strongest reflections are shown (full pattern: see Ref. [14]). The calculated x-ray patterns corresponding to simulations at $70 \mathrm{GPa}$, below and above the transition temperature to the superionic phase, are shown below. The inset is a zoom on the (120) reflection, which is observed in phase $\mathrm{V}$ but is absent in the $\alpha$-superionic phase. The (hkl) refer to the orthorhombic structure. (c) Lattice parameters along the $70.6 \mathrm{GPa}$ isobar. (d) Volume (left axis) and entropy (right axis) variation along the order-disorder transition line. Symbols are experimental data, and lines are guides to the eye. The error bars for $\Delta S$ are mostly influenced by the precision on the slope of the transition line accessible from our data. (e) Isothermal compressibility modulus at $750 \mathrm{~K}$. Dashed lines are guides to the eye to emphasize the change at $\sim 55 \mathrm{GPa}$.

$3.8 \times 10^{-5} \mathrm{~cm}^{2} / \mathrm{s}$ in liquid ammonia [17] and $(1-1.17) \times 10^{-7} \mathrm{~cm}^{2} / \mathrm{s}$ in the superprotonic phase of $\mathrm{CsHSO}_{4}$ [18]. The high $P-T$ phase thus clearly qualifies as a superionic conductor.

Annealing phase V at $100 \mathrm{GPa}$ reveals a similar evolution towards the superionic phase. Some " $c$ jumps" are, however, observed at a lower temperature $(450 \mathrm{~K})$, while " $a b$ jumps" start above $\sim 600 \mathrm{~K}$. Here again, proton diffusion across the crystal is correlated with proton jumps within the molecular planes and increases rapidly above $\sim 750 \mathrm{~K}$. We also simulated the isothermal compression of the low-pressure hcp and fcc plastic phases, at 900 and $850 \mathrm{~K}$, respectively. In both cases, fast proton diffusion is observed at $P>50 \mathrm{GPa}$ in good correspondence with the experimental pressure $(55 \mathrm{GPa})$ where we observe the sudden change of compressibility at $750 \mathrm{~K}$. The superionic phase thus exists whether the $N$ lattice is hcp or fcc. This allows us to draw a tentative transition line between the plastic and superionic solids as shown in Fig. 1. The absence of measurable volume discontinuity or a change of lattice symmetry at the plastic-superionic transition may indicate that it is of type II (i.e., continuous), whereas the transition between the ordered and superionic solids is of type I (first-order).

Our simulations suggest that the larger structural effect of the transition to the superionic phase should be seen along the crystallographic $c$ axis, as the number of proton jumps increases much more rapidly along $\mathrm{HB}_{c}$ bonds than $\mathrm{HB}_{a b}$ bonds at the onset of superionic conduction. This is precisely what is observed at the $\mathrm{V}-\alpha$ phase transition in our experiments, the larger structural effect being the discontinuity of the $c$ axis length. This anisotropy is likely related to the fact that the $\mathrm{HB}_{c}$ bonds are stronger than the $\mathrm{HB}_{a b}$ ones, as they are shorter and become linear at the IV-V transition [11,12]. The very good match between the experimental and theoretical transition temperatures, for both the ordered-plastic transition line and the molecular-superionic transition, also gives good confidence in assigning the $\alpha$ phase to the superionic one. Another strong evidence comes from the comparison between the experimental x-ray pattern of the $\alpha$ phase and the one of the simulated superionic phase at the same pressure: 

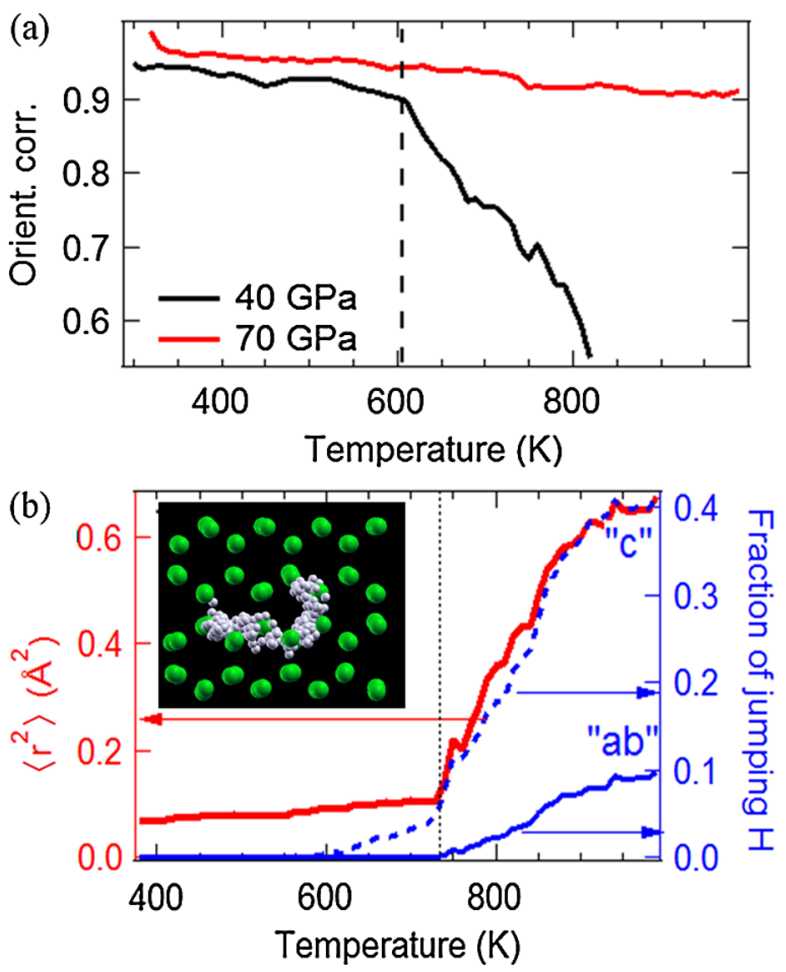

FIG. 3 (color online). (a) Orientational correlations of $\mathrm{NH}_{3}$ molecules in the crystal as function of $T$ (see Theoretical Methods in Ref. [14]). The order-plastic transition occurs near $600 \mathrm{~K}$ at $40 \mathrm{GPa}$ (black curve). By contrast, no sign of plasticity is observed at $70 \mathrm{GPa}$ up to $1000 \mathrm{~K}$ (red curve). (b) Diffusion (left axis) and fraction of proton jumps (right axis) as a function of $T$ at $70 \mathrm{GPa}$. Solid and dotted blue lines: Jumps along $\mathrm{HB}_{c}$ and $\mathrm{HB}_{a b}$, respectively. Red curve: Mean square displacement of $\mathrm{H}$ atoms with respect to their initial positions. Proton diffusion sets in at $740 \mathrm{~K}$. Inset: Illustration of diffusion of one $\mathrm{H}$ atom (gray) at $70 \mathrm{GPa}$ and $850 \mathrm{~K}$ in the fixed lattice of (green) $N$ atoms. The 100 different positions of the $\mathrm{H}$ atom are time-spaced by $40 \mathrm{fs}$.

They are very similar, as can be seen in Fig. 2(b). In particular, the extinction of the (120) reflection, whose intensity is strongly correlated with the positions of the $\mathrm{H}$ atoms, is predicted in the superionic phase and clearly observed at the V- $\alpha$ phase transition. The large increase of entropy at the $\mathrm{V}-\alpha$ transition is also well explained by the appearance of ionic species in the superionic phase predicted by our simulations. To demonstrate this, we evaluate the configurational entropy of the ordered and disordered phases by counting the number of accessible sites for the $\mathrm{H}$ atoms using a simple model whose details are presented in Ref. [14]. We obtain a variation of the configurational entropy of $0.91 R$ if only orientational disorder is taken into account, which agrees very well with our measured value $[1.0(4) R]$ at the order-plastic transition below $57 \mathrm{GPa}$ [Fig. 2(d)]. When the creation of ionic species is included in the model, the configurational entropy increases up to $2.24 R$, which again favorably compares with the entropy variation measured at the $\mathrm{V}-\alpha$ transition $(4 R \pm 2 R)$. We note that this model neglects the additional vibrational entropy coming from the vibrational modes of the $\mathrm{NH}_{4}^{+}$or $\mathrm{NH}_{2}^{-}$ions, which should make the calculated entropy variation in even closer agreement with the experimental one. All these observations strongly suggest that the experimental V- $\alpha$ transition line coincides with the onset of superionic conduction.

The present results thus validate the prediction [2] of a superionic ammonia ice but locate it at lower $T(\sim 750 \mathrm{~K}$ instead of $\sim 1200 \mathrm{~K}$ ). This value is also significantly lower than the ones found in water ice at the ice VII-superionic phase transition [19] (1500 K at $50 \mathrm{GPa})$. This result should be relevant for the stability of the superionic phase in the $\mathrm{H}_{2} \mathrm{O}-\mathrm{CH}_{4}-\mathrm{NH}_{3}$ mixtures of the Jovian planets. Furthermore, the strong lowering with pressure of the temperature at which $\left(\mathrm{NH}_{4}^{+}, \mathrm{NH}_{2}^{-}\right)$pairs are formed by proton jumps along $\mathrm{HB}_{c}$ bonds is consistent with the theoretically predicted layered ionic phase [1] at low $T$. Our results suggest that the anisotropy of the $\mathrm{H}$ bonds in the ordered phase of $\mathrm{NH}_{3}$ ice drives the formation of this ionic phase at high density, whereas a symmetrization of the $\mathrm{H}$ bonds is observed in water ice where all $\mathrm{H}$ bonds are equivalent. The existence of this ionic phase remains to be experimentally confirmed, and its link with the superionic state reported here should motivate further studies.

We acknowledge the ESRF for beam time (HS3937) and the IDRIS Supercomputing Facility for CPU time (CP9101387). We thank M. Hanfland (ID09) and P. Munsch for their help with X-ray experiments. A. M.S. thanks the French Agence Nationale de la Recherche (ANR) for support through Project JCJC-HPQUENS (2009-2013).

[1] C. J. Pickard and R. J. Needs, Nature Mater. 7, 775 (2008).

[2] C. Cavazzoni, G. L. Chiarotti, S. Scandolo, E. Tosatti, M. Bernasconi, and M. Parrinello, Science 283, 44 (1999).

[3] A. F. Goncharov, N. Goldman, L. E. Fried, J. C. Crowhurst, I.-F. W. Kuo, C. J. Mundy, and J. M. Zaug, Phys. Rev. Lett. 94, 125508 (2005).

[4] B. Schwager, L. Chudinovskikh, A. Gavriliuk, and R. Boehler, J. Phys. Condens. Matter 16, S1177 (2004).

[5] J.-F. Lin, E. Gregoryanz, V. V. Struzhkin, M. Somoayazulu, H.-K. Mao, and R.J. Hemley, Geophys. Res. Lett. 32, L11 306 (2005).

[6] W. J. Nellis, D.C. Hamilton, N.C. Holmes, H. B. Radousky, F.H. Ree, A.C. Mitchell, and M. Nicol, Science 240, 779 (1988).

[7] H. Radousky, A. Mitchell, and W. Nellis, J. Chem. Phys. 93, 8235 (1990).

[8] W. J. Nellis, N. C. Holmes, A. C. Mitchell, D. C. Hamilton, and M. Nicol, J. Chem. Phys. 107, 9096 (1997).

[9] J. S. Loveday, R. J. Nelmes, W. G. Marshall, J. M. Besson, S. Klotz, and G. Hamel, Phys. Rev. Lett. 76, 74 (1996). 
[10] S. Ninet, F. Datchi, A. M. Saitta, M. Lazzeri, and B. Canny, Phys. Rev. B 74, 104101 (2006).

[11] S. Ninet, F. Datchi, S. Klotz, G. Hamel, J. S. Loveday, and R. J. Nelmes, Phys. Rev. B 79, 100101(R) (2009).

[12] A. D. Fortes, J. P. Brodholt, I. G. Wood, and L. Vočadlo, J. Chem. Phys. 118, 5987 (2003).

[13] F. Datchi, S. Ninet, M. Gauthier, A. M. Saitta, B. Canny, and F. Decremps, Phys. Rev. B 73, 174111 (2006).

[14] See Supplemental Material at http://link.aps.org/ supplemental/10.1103/PhysRevLett.108.165702 for auxiliary information on experimental methods, experimental refinement of the x-ray pattern, the entropy variation model, and theoretical methods.

[15] S. Ninet and F. Datchi, J. Chem. Phys. 128, 154508 (2008).

[16] N. Agmon, Chem. Phys. Lett. 244, 456 (1995).

[17] A. Goncalves, C. Mathieu, M. Herlem, and A. Etcheberry, J. Electroanal. Chem. 477, 140 (1999).

[18] A. V. Belushkin, C. J. Carlile, and L.A. Shuvalov, Ferroelectrics 167, 21 (1995).

[19] E. Schwegler, M. Sharma, F. Gygi, and G. Galli, Proc. Natl. Acad. Sci. U.S.A. 105, 14779 (2008). 